\title{
Defect Fruit Image Analysis using Advanced Bacterial Foraging Optimizing Algorithm
}

\author{
P.Lakshmi Devi ${ }^{1}$ S.Varadarajan ${ }^{2}$ \\ 1. Associate Professor, AITS, Rajampet, KADAPA, AP. India \\ 2. Professor, SVUCE, Thitupathi, India AP.
}

\begin{abstract}
Bacterial foraging optimization algorithm has been widely accepted as a global optimization algorithm. Since Image segmentation is the basic step in many image processing applications, so faithful segmentation algorithm must be developed for successful implementation of the processing applications. Core aim of image segmentation is to extract the information which is of interest for a particular application. Infact this research addresses the novel segmentation of infected part of fruits based on color features and the image into various color components by using combined approach of Advanced Bacterial foraging optimization Algorithm (ABFOA) approach and RGB decomposition. The original image is decomposed into separate planes of $R G$ and $B$ and then Improved Bacterial Foraging algorithm is applied on three planes separately to calculate three different thresholds. The experimental results clarify the effectiveness of proposed approach to improve the segmentation quality in aspects of precision and computational time. The simulation results reveal that the proposed approach is promising. Segmentation is performed on the basis of Thresholding.
\end{abstract}

Key Words: Improved Bacterial Foraging Optimization Algorithm, Segmentation, Fruit Images, Image Processing.

\section{Introduction}

Extracting the information from images and understanding them for several tasks is an important characteristic of Machine learning. Also, image segmentation is one of the initial steps in direction of understanding images and then finds the different objects in them. As modern agricultural science and technology is in extreme advance, it is an important issue how to examine quality of fruit in agricultural science and technology. The value of fruit depends on the quality of fruit. The classical approach of fruits quality assessment is done by the experts and it is very time consuming. Image segmentation of the image is nothing but pixel classification. The difficulty to which the image segmentation process is to be carried out mostly depends on the particular problem that is being solved. It is one of the most crucial components of image analysis and pattern recognition and still is considered as most challenging tasks for the image processing and image analysis. Image segmentation methods are generally based on one of two fundamental properties of the intensity values of image pixels: similarity and discontinuity. In the first category, the concept is to partition the image into several different regions such that the image pixels belonging to a region are similar according to a set of predefined criteria's. Whereas, in the second category, the concept of partition an image on the basis of abrupt changes in the intensity values is used. Also, Color is a perceptual phenomenon related to human response to different wavelengths in the visible electromagnetic spectrum and color is the most prominent feature of any image. Extracting color information from any image has many applications related to computer vision algorithms. Color of an image can carry much more information than gray level and for an instance of the image segmentation in which this research $\mathrm{h}$ addresses only to the infected portion of the fruit.

\section{Summary Of The Related Work}

The control system of these bacteria that dictates how foraging continue and consists of four principal mechanisms, namely chemotaxis, swarming, reproduction, and elimination dispersal and the four basic steps in BFOA are explained below Sambarta Dasgupta et.al 2009.

a) Chemotaxis:- In the original BFO, a unit walk of the bacteria with random direction represents a "tumble" and a unit walk with the same direction in the last step indicates a run. It can move in two different strategies. It can swim for a period of time in the same direction or it may tumble, and alternate between these two modes of operation for the entire lifetime.

b) Swarming: - A group of E.coli cells arrange themselves in a travelling ring by moving up the nutrient gradient when placed amidst a semisolid matrix with a single nutrient chemo-effecter. The cells when stimulated by a high level of succinate, release an attractant aspartate, which helps them to aggregate into groups and thus move as concentric patterns of swarms with high bacterial density. 
c) Reproduction:- The least healthy bacteria eventually die while each of the healthier bacteria (those yielding lower value of the objective function) asexually split into two bacteria, which are then placed in the same location. This keeps the population of bacteria constant.

d) Elimination and Dispersal: - The chemotaxis provides a basis for searching the local best solution and reproduction process speeds up the convergence which has been simulated by the classical BFO. The bacteria with the best positions are kept and the remaining bacteria population is killed. The bacteria with best positions are then moved to another position within the environment.

\subsection{Colour Image Segmentation}

Color image segmentation has been a difficult task for the researchers over the past two decades. It is an essential operation in image processing and in many computer vision, pattern recognition, and image interpretation system Silva RD et.al 2008. The efficiency of color image segmentation may significantly influence the quality of an image understanding system $H C$ Chen et.a 2006l. A detail review on various image segmentation techniques are provided by N R Pal et.a 1993l. The main characteristics used as the basis of color image segmentation are color and texture. Color histograms are commonly used in content-based retrieval systems and have proven to be very useful. Various different color spaces have been defined which simply described the colors, or gamut that a camera can see, a printer can print or a monitor can display. The allocation of individual pixels into heir corresponding segments based on the color changes largely aims at maximizing the total information and minimizing the differences between the amounts of information contained in the different segments Beenu et.al 2013. Soft computing techniques have been used for segmenting color image by Sowmya et.al 2009. The soft computing techniques they used were competitive neural network and Possibilistic C means algorithm (PCM). Researchers also used Fuzzy set and Fussy logic techniques for solving segmentation problem. Borji et al. presented CLPSO-based Fuzzy color image segmentation Borli A et.al, 2007. Cheng et al. used Fuzzy homogeneity approach for the segmentation of color image Cheng HD et.al 1998. Besides this, Genetic algorithm (GA) and artificial neural network (ANN) techniques also have been used for the image segmentation Birgani PM et.al, 2008.

\section{Proposed Methodology}

An important goal of image segmentation is to separate the object and background clear regardless the image has blur boundary. Defect segmentation of fruits can be seen as an instance of image segmentation in which number of segmentation is not clearly known. The proposed improved bacterial foraging algorithm is implemented on the input image in the following steps:

\subsection{RGB Decomposition}

Step-1: The input image is first converted into its RGB image components to get three images in red, green and blue components.

Step-2: Maximum No. of Colors in any color component image $=256$ (Black to Red, Black to Green and Black to Blue)

Step-3: Initialize Threshold in each RGB component image as $\mathrm{TR}=0, \mathrm{TG}=0$ and $\mathrm{TB}=0$

Step-4: Take the red component image. Compute the size of the image into row $\mathrm{x}$ column giving rise to bacterial search area.

Step-5: Initialize the $\mathrm{Nc}$ and $\mathrm{Ns}$ steps at $1 . \mathrm{Nc}=1$ and $\mathrm{Ns}=1$; Chemo tactic and swim length.

Step-6: Compute the health status of all image pixels by using the image histogram. The health status of $\mathrm{i}^{\text {th }}$ color pixel $\mathrm{Hi}$ is given by: $\mathrm{Hi}=\mathrm{Pi}$ / (row $\mathrm{x}$ column), where $\mathrm{Pi}$ is the no. of pixels of $\mathrm{i}^{\text {th }}$ color.

Step-7: Compute the Euclidean distance ED between the adjacent pixels as ED $=F(r, c)-F(r, c+1)$ Where $F(r, c)$ and $\mathrm{F}(\mathrm{r}, \mathrm{c}+1)$ are the pixel color value of two adjacent pixels.

Step-8: If ED is less than some threshold ED, then replace the F(r,c) by F(r,c+1), thereby reducing the no. of colors in the entire image.

Step-9: Now compute the difference of health status of $\mathrm{H}(\mathrm{r}, \mathrm{c})$ and $\mathrm{H}(\mathrm{r}, \mathrm{c}+1)$ pixel . If $\mathrm{H}(\mathrm{r}, \mathrm{c})$ and $\mathrm{H}(\mathrm{r}, \mathrm{c}+1)$ are less than the threshold health status, then they are the unpopular colors and can be eliminated to produce a new color.

Step-10:Keep on adding the color value to TH, TG or TR as may be the case from step-4.

Step-11:Move the pixel pointer over the entire image.

Step-12:Repeat from step-4 to 11 for green and blue component images in the same manner and compute the TR, TG TB.

Step-13:Compute the individual thresholds as given by: $\mathrm{TH}=\mathrm{TH} / \mathrm{Image}$ Size, $\mathrm{TG}=\mathrm{TG} / \mathrm{Image}$ Size $\mathrm{TB}=$ TB/Image Size

Step-14:Compute the final threshold as given by: $T=(T H+T G+T R) / 3$ 
Step-15:Apply the final threshold over the original image and compute the performance indices as standard deviation, entropy, PSNR.

\subsection{Objective function for $\mathrm{BFOA}$}

The proposed objective function is given below:

Let, the No. of Threshold Level

$$
\begin{aligned}
& \mathrm{K}=\left\{\mathrm{t}_{1}, \mathrm{t}_{2}, \mathrm{t}_{3}, \ldots \mathrm{t}_{\mathrm{K}}\right\}, \\
& \text { Then the objective function } H \text { is given by: }
\end{aligned}
$$$$
H_{k}=\sum_{i=t k}^{2-1} \frac{P i}{\omega k} \ln \left(\frac{P i}{\omega k}\right)
$$$$
\text { Where } \omega_{k}=\sum_{i=t h}^{L-1} P i
$$

And Pi is the histogram value of the $\mathrm{i}^{\text {th }}$ gray level.

The proposed objective function is a global objective function based on entropy in combination with histogram, and the user can tailor the objective function based on the application. If the no. of threshold levels is 2 , then the system becomes binary thresholding based on Otsu method. However, the same algorithm can be extended to multi-level thresholding if the value of $\mathrm{K}$ is more than 2. Later, the maxima of the selected threshold is optimized by using the BFO algorithm based on chemotaxis with random value of length within limit, random rate of elimination and dispersion of bacteria and random swim and tumbling of bacteria. The random rate of swim, tumbling and rate of elimination and dispersion give a better optimization of the maxima of the threshold level from the given threshold levels.

The movement of the ith bacterium is described by

$$
\begin{aligned}
& \text { Ps }(f+1, g ; u)=\text { Ps }(f ; g ; u)+C(s) \text { x V (f) } \\
& \text { Where Ps (f; g; } u \text { ) is the } \mathrm{s}^{\text {th }} \text { location of the bacterium at the } \\
& \mathrm{f}^{\text {th }} \text { Chemotactic, } \mathrm{g}^{\text {th }} \text { reproductive, and } \mathrm{u}^{\text {th }} \text { elimination steps. }
\end{aligned}
$$

$\mathrm{C}(\mathrm{s})$ is the length of one walking cycle. It is defined as a small constant value. $\mathrm{V}(\mathrm{f})$ is the direction angle of the $\mathrm{f}^{\text {th }}$ chemotactic step; its default value is set at a range of $[0 ; 2 \pi]$.

In the Advanced BFOA random reproduction and elimination of the bacterium is added so that the more natural randomness could be added in order to enhance the segmentation process. The random reproduction and elimination is inserted Mat-Lab programming. The 'for' loop is executed in random manner where the incremental operator is incremented by some random number.

\subsection{Performance Measures}

In order to evaluate and compare the resultant threshold image with proposed algorithm with respect to other algorithms, following performance measures are suggested:

a) PSNR: The peak-signal to noise ratio (PSNR) was used to evaluate the reconstructed image quality. The PSNR is defined as follows:

$$
P S N R=10 \log _{10} \frac{255^{2}}{\frac{1}{N \times N} \sum_{i=0}^{N-1} \sum_{j=0}^{N-1}(f(i, j)-\hat{f}(i, j))^{2}} d B,
$$

Where $\mathrm{N} \times \mathrm{N}$ is the size of the original image and $f(i, j)$ and ${ }^{\wedge}(\mathrm{i}, \mathrm{j})$ are the gray-level pixel values of the original and reconstructed images, respectively.

b) Standard Deviation (SD): The standard variation of an image is given by:

$$
\hat{\sigma}^{2}=\frac{1}{n \times n} \sum_{j=1}^{n} \sum_{i=1}^{m}\left(x_{i, j}-\hat{\mu}\right)^{2},
$$

This corresponds to the degree of deviation between the gray levels and its mean value, for the overall image.

c) Entropy E: The expression of the information entropy of an image is given by:

$$
\mathrm{H}=-\sum_{i=0}^{L-1} P i \ln P i
$$

Where $\mathrm{L}$ denotes the number of gray level, pi equals the ratio between the number of pixels whose gray value equals $\mathrm{i}(0$ to $\mathrm{L}-1)$ and the total pixel number contained in an image. The information entropy measures the richness of information in an image. If pi is the constant for an arbitrary gray level, it can be proved that the entropy will reach its maximum. 
d) Class Variance: Class variance of the segmented image is computed by the following computation method: If the histogram is divided into two classes by the gray-level intensity $t$ (threshold), then the probabilities of the respective classes can be expressed as:

$$
\begin{aligned}
& \mathrm{P}_{1}(\mathrm{t})=\sum_{i=0}^{t} p(i) \quad \text { and } \mathrm{P}_{2}(\mathrm{t})=\sum_{i=t+1}^{N-1} p(i) \text { Also, the class means } \mathrm{m}_{1} \text { and } \mathrm{m}_{2} \text { are given by: } \\
& m 1(t)=\sum_{i=0}^{t} i p(i) / p 1(t) \\
& m 2(t)=\sum_{i=t+1}^{N-1} i p(i) / p 2(t)
\end{aligned}
$$

The two class variances are given by:

$$
\begin{aligned}
& \sigma_{1}^{2}(\mathrm{t})=\sum_{i=0}^{t}(i-m 1)(i-m 1) p i / p 1(t) \\
& \sigma_{2}{ }^{2}(\mathrm{t})=\sum_{i=t+1}^{N-1}(i-m 2)(i-m 2) p i / p 2(t)
\end{aligned}
$$

The total class variance $\left(\sigma_{\mathrm{T}}\right)$ is given by: $\quad \sigma_{\mathrm{T}}{ }^{2}=\sigma_{\mathrm{B}}{ }^{2}+\sigma_{\mathrm{w}}{ }^{2}$

Where $\sigma_{\mathrm{B}}{ }^{2}$ is the between class variance and $\sigma_{\mathrm{w}}{ }^{2}$ within class variance and given by following equations.

$$
\sigma_{\mathrm{w}}^{2}(\mathrm{t})=\mathrm{P}_{1}(\mathrm{t}) \sigma_{1}^{2}(\mathrm{t})+\mathrm{P}_{2}(\mathrm{t}) \sigma_{2}^{2}(\mathrm{t})
$$

$$
\begin{aligned}
& \sigma_{\mathrm{B}}^{2}(\mathrm{t})=\mathrm{P}_{1}(\mathrm{t}) . \mathrm{P}_{2}(\mathrm{t})\left\{\mathrm{m}_{1}(\mathrm{t})-\mathrm{m}_{2}(\mathrm{t})\right\}^{2} \text { and the Flow Chart for the proposed infected } \\
& \text { fruit image Segmentation is given in Figure.1. }
\end{aligned}
$$

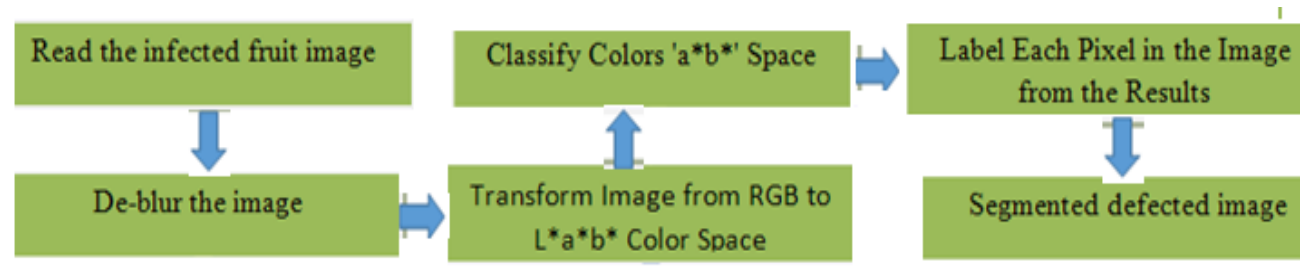

Fig.3.1: Flow Chart for the proposed infected fruit image Segmentation

\section{Results And Discussion}

To demonstrate the performance of the proposed approach, the initial images of fruits are depicted from Shivaram Duby et.al 2013, apples such as apple scab, apple rot, apple dieses and apple blotch for the defect segmentation. The figures 2, 3, 4 and figure. 5 show segmentation results using proposed approach of RGB decomposition and ABFOA. Proposed algorithm offers a more resolved thresholding in comparison to any other thresholding method like Otsu.

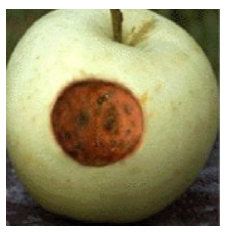

(a)

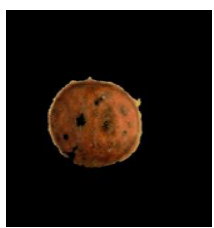

(b)

Fig.2: Scab Apple (a) Before segmentation

(b) After Segmentation

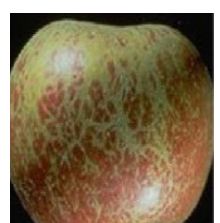

(a)

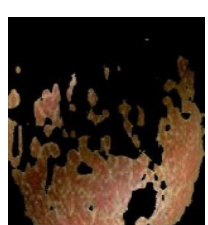

(b)

Fig.4: Infected Apple (a) Before segmentation segmentation

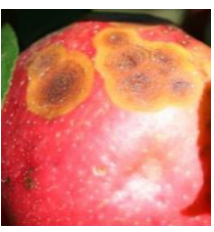

(a)

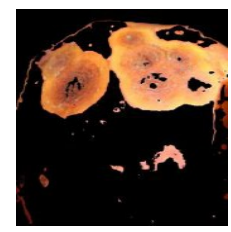

(b)
Fig.3: Rot Apple (a) Before segmentation

(b) After Segmentation

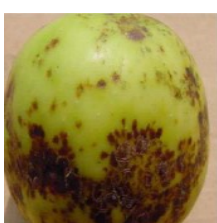

(a)

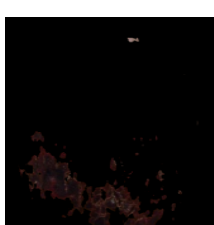

(b)
Fig.5: Blotch Disease Apple (a) Before (b) After Segmentation

(b) After Segmentation 
The image decomposition into its RGB components and computation of threshold in each component image using ABFOA algorithm prove to be a good tool in order to faithfully segment or threshold the image The experimental results suggest that the proposed approach is able to accurately segment the defected area of fruits present in the image. Threshold, entropy, Standard Deviation, and PSNR is being calculated and shown in Table.1. The performance indices calculated and the results are better than the previously suggested technique Otsu.

\section{Collusion}

As all the optimization algorithms are stochastic and random searching one, the results of experiments are not absolutely the same in each run of the algorithm. One important concern in image segmentation is the effectiveness in thresholding. According to the segmented results, the proposed ABFOA method has demonstrated satisfactory results. However, it is somewhat difficult to compare quantitatively the performance of global thresholding results. Three common performance evaluation criteria, the Peak to Signal Noise Ratio (PSNR), entropy and standard deviation measure, are employed to evaluate the thresholding methods. The future work includes automatic determination of number of clusters required to segment the defects more accurately.

Table.1: Entropy:: Threshold::Standard Deviation::PSNR Comparison between Otsu and ABFOA

\begin{tabular}{|c|c|c|c|c|c|c|c|c|c|}
\hline \multirow[t]{2}{*}{ Image } & \multirow[t]{2}{*}{$\begin{array}{l}\text { Image } \\
\text { Size }\end{array}$} & \multicolumn{2}{|c|}{ ENTROPY } & \multicolumn{2}{|c|}{ THRESHOLD } & \multicolumn{2}{|c|}{$\begin{array}{l}\text { STANDARD } \\
\text { DEVIATION } \\
\end{array}$} & \multicolumn{2}{|c|}{ PSNR } \\
\hline & & Otsu & $A B F O A$ & Otsu & $A B F O A$ & Otsu & $A B F O A$ & Otsu & $A B F O A$ \\
\hline \multirow{3}{*}{$\begin{array}{l}\text { Scab } \\
\text { Apple }\end{array}$} & $512 \times 512$ & 0.9478 & 0.9976 & 0.4546 & 0.4701 & 0.4989 & 0.4365 & 58.875 & 69.784 \\
\hline & $256 \times 256$ & 0.9245 & 0.9642 & 0.4551 & 0.4699 & 0.4628 & 0.4145 & 62.855 & 70.047 \\
\hline & $96 \times 96$ & 0.8992 & 0.9437 & 0.4539 & 0.4652 & 0.4532 & 0.4101 & 65.477 & 70.011 \\
\hline \multirow{3}{*}{$\begin{array}{c}\text { Rot } \\
\text { Apple }\end{array}$} & $512 \times 512$ & 0.9667 & 0.9988 & 0.4824 & 0.4988 & 0.4783 & 0.3998 & 65.984 & 71.385 \\
\hline & $256 \times 256$ & 0.9567 & 0.9867 & 0.4645 & 0.4848 & 0.4576 & 0.4143 & 64.547 & 70.375 \\
\hline & $96 \times 96$ & 0.9789 & 0.9889 & 0.4611 & 0.4840 & 0.4334 & 0.4011 & 68.984 & 71,012 \\
\hline \multirow{3}{*}{$\begin{array}{l}\text { Infected } \\
\text { Apple }\end{array}$} & $512 \times 512$ & 0.8798 & 0.9134 & 0.4124 & 0.4424 & 0.4789 & 0.4094 & 69.855 & 68.857 \\
\hline & $256 \times 256$ & 0.9012 & 0.9465 & 0.4111 & 0.4323 & 0.4678 & 0.4992 & 65.844 & 67.439 \\
\hline & $96 \times 96$ & 0.9014 & 0.9562 & 0.4024 & 0.4311 & 0.4821 & 0.4857 & 61.987 & 68.745 \\
\hline \multirow{3}{*}{$\begin{array}{l}\text { Blotch } \\
\text { Apple }\end{array}$} & $512 \times 512$ & 0.9273 & 0.9836 & 0.4303 & 0.4822 & 0.4986 & 0.4089 & 59.897 & 65.987 \\
\hline & $256 \times 256$ & 0.9267 & 0.9134 & 0.4219 & 0.4673 & 0.4897 & 0.3758 & 63.985 & 67.983 \\
\hline & $96 \times 96$ & 0.9285 & 0.9482 & 0.4356 & 0.4867 & 0.4894 & 0.4184 & 67.947 & 69.987 \\
\hline
\end{tabular}

\section{References}

[1]. Beenu, Sukhwinder Kaur "Image Segmentation using Improved Bacterial Foraging Algorithm" International Journal of Science and Research (IJSR)-2013

[2]. Birgani PM , M. Ashtiyani, and S. Asadi, "MRI segmentation using fuzzy c-means clustering algorithm basis neural network," in Proceedings of the 3rd International Conference on Information and Communication Technologies: From Theory to Applications, 2008, pp. 1-5.

[3]. Borji A, M. Hamidi, and A. M. E. Moghadam, "CLPSO-based fuzzy color image segmentation," in Proceedings of the North American Fuzzy Information Processing Society, 2007, pp. 508-513.

[4]. Chen HC and S. J. Wang, "Visible color difference-based quantitative evaluation of colour segmentation," in Proceedings of the IEEE Conference on Vision Image and Signal processing, 2006, vol. 153, no. 5, pp. 598-609.

[5]. Cheng H D, C. H. Chen, H. H. Chiu, and H. Xu, "Fuzzy homogeneity approach to multilevel thresholding," IEEE Transactions On Image Processing (TIP), vol. 7, no. 7, pp. 1084-1086, 1998.

[6]. Pal NR and S. K. Pal, “A review on image segmentation techniques,” Pattern Recognition, vol. 26, no. 9, pp. 1227-1294, 1993.

[7]. Sambarta Dasgupta, Swagatam Das "Bacterial Foraging Optimization Algorithm: Theoretical Foundations, Analysis, and Applications" Studies in Computational intelligence volume 203,2009 pp23-55.

[8]. Shiv Ram Dubey, Pushkar Dixit, Nishant Singh, Jay Prakash Gupta " Infected fruit part detection using K-means clustering segmentation technique" International Journal of Artificial Intelligence and Interactive Multimedia, 2013 Vol. $2, N^{\circ} 2$.

[9]. Silva RD, R. Minetto, W. R. Schwartz, and H. Pedrini, "Satellite image segmentation using wavelet transforms based on color and texture features," in Proceedings of the 4th International Symposium on Advances in Visual Computing, Part II, 2008, pp. 113-122.

[10]. Sowmya Band B. Sheelarani, "Colour image segmentation using soft computing techniques," International Journal of Soft Computing Applications, vol. 4, pp. 69-80, 2009.

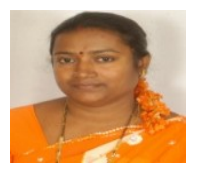

Mrs Lakshmi Devi is graduated from SVU College of engineering Thirupathi and obtained Masters Degree from JNTU Anantapur, Andhra Pradesh. Currently she is working as an Associate Professor at AITS, Rajampet for the last 14 years. Her research interests includes Signal Processing, Image Processing, Evolutionary Algorithms etc,.

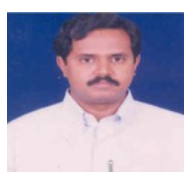

Dr Souri Varadarajan is an eminent professor in the field of Electronics and Communications Engineering, having about 20 years of teaching experience and currently working at SV University college of Engineering Thirupathi, Andhra Pradesh, India. His research interests includes Digital Image Processing, Image processing, Signal Processing etc, 